\title{
LA GUERRA COMO CUESTIÓN SOCIAL: UN ACERCAMIENTO A LA POLÍTICA SOCIAL COLOMBIANA
}

\author{
WAR AS A SOCIAL ISSUE: AN APPROACH TO COLOMBIAN \\ SOCIAL POLICY
}

\section{Daniel Castaño Zapata*}

\begin{abstract}
RESUMEN
El objetivo de este artículo es dejar planteadas algunas líneas para pensar la relación entre la política social en Colombia y la guerra interna, que se desarrolla en este país desde mediados del siglo xx. Para ello se realiza una presentación del enfoque conceptual desde el que se propone analizar las principales dimensiones, teóricas y empíricas, en que las políticas sociales colombianas son construidas por la guerra.
\end{abstract}

PALABRAS CLAVE: COLOMBIA * POLÍTICA SOCIAL * GUERRA * CUESTIÓN SOCIAL

\section{ABSTRACT}

The aim of this article is to make some lines raised to think the relationship between social policy in Colombia and internal war, which takes place in this country since the middle of xx's Century. For this study, it makes a presentation from the conceptual approach proposed to be the main dimensions, theoretical and empirical, that Colombian social policies are built by war.

KEYWORDS: COLOMBIA * SOCIAL POLICY * WAR * SOCIAL ISSUE

Grupo de Estudios sobre Políticas Sociales y Condiciones de Trabajo del Instituto Gino Germani, Universidad de Buenos Aires, Argentina (UBA).

dacazap@gmail.com 


\section{INTRODUCCIÓN}

La hipótesis que guía este desarrollo puede ser planteada de la siguiente manera: la formulación e implementación de buena parte de las políticas sociales en Colombia, por lo menos de las últimas cuatro décadas, las cuales han sido producto de las distintas tensiones $y$ diferentes "racionalidades" sociales construidas a la par de la dinámica de su guerra interna. Se sostiene que el fenómeno de la guerra construye la política social colombiana en cuatro dimensiones: a) radicalizando su participación en la construcción de la sociedad como ordenamiento simbólico; b) interviniendo en el proceso de definición de sus actores; c) sus problemas $y$ d) su agenda.

Antes de ingresar específicamente en el tema, es importante establecer algunas precisiones conceptuales que ordenan la discusión. Concretamente se propone un acercamiento, con la pretensión de un mínimo consenso, a definiciones problemáticas como: guerra, política social, merecimiento y cuestión social.

En este artículo se entenderá y nombrará la problemática del enfrentamiento de grupos armados (legales e ilegales) en Colombia, a partir del concepto "Guerra", valorado como un núcleo común de significación que abarca distintas manifestaciones y tipologías que designan los enfrentamientos armados. Por eso, se propone retomar la definición de "guerra" que realiza Bobbio (1992) y a partir de ella, abordar $y$ describir las características de la relación presentada como problema de estudio.

Bobbio propone comprender la guerra como un conflicto entre grupos políticos, respectivamente independientes o considerados tales, cuya solución se confía a la violencia organizada (1992: 162). Esta definición obliga a pensar la guerra a partir de dos elementos fundamentales que subyacen en la complejidad, no solo de las discusiones sobre política en general, sino sobre la política social en particular y específicamente, la política social colombiana: en primer lugar, se deben considerar los actores armados como grupos políticos, esto es, "organizados para el mantenimiento o la conquista del máximo poder posible entre y sobre hombres que conviven" (Bobbio, 1992: 163) y en segundo lugar, se debe entender la violencia organizada como un género de violencia instrumentado para garantizar el dominio de hecho sobre un territorio de modo permanente, con el fin de obtener obediencia a las propias ordenes por parte de la población'1.

Comprender la guerra en Colombia, a partir de una definición general como la anterior, permite pensar de manera más amplia las intersecciones entre esta $y$ la disciplina teórica que motiva este análisis: la política social.

Es importante tener presente que, cuando se busca indagar en la historia de la política social de un Estado nacional, generalmente, las preguntas giran en torno a los modos en cómo en esa sociedad, la política social participa de la producción de lo que se denomina "el mundo del trabajo" y de lo que a él se sustrae "mundo de la vida". En este sentido, la pregunta por la política social indaga tanto por las condiciones a partir de las cuales los sujetos "con obligación $y$ necesidad" de trabajar cumplen el mandato del autovalimiento, como por las características que deben tener los sujetos que dependen de la asistencia y sus condiciones de integración social, así como, por las tensiones sociales e históricas que construyen una idea de cuestión social.

En este sentido, en lo que respecta al vector "sujetos", el "merecimiento" es una de las categorías fundamentales de los estudios que abordan las políticas sociales desde el análisis de las condiciones de trabajo, pues dispone las razones que construyen al grupo de sujetos que son destinatarios de las distintas intervenciones sociales del Estado. Una característica que define a los miembros de estos grupos es que deben ante todo ( $y$ ante todos o al menos ante la mayoría) cumplir ciertas condiciones para "merecer". Siendo las razones más clásicas del merecimiento aquellas que se fundan

1 Esta idea de apelar a un núcleo común de significación como estrategia epistemológica y metodológica, si bien pertenece a Bobbio, fue tomada del trabajo de Giraldo (2009), quien la desarrolla a profundidad. En dicho trabajo, se señala además que, una definición aún mas amplia que la de Bobbio respecto del significante guerra, es la aportada por Bouthol, quien define la guerra como "una lucha armada y sangrienta entre agrupaciones organizadas" (Bouthol, 1971: 35). 
en la incapacidad (enfermos, inválidos), en la adjudicación de responsabilidades domésticas (mujeres), en la necesidad de un tiempo de aplazamiento y capacitación antes de participar directamente en actividades productivas (niños o jóvenes) y en el reconocimiento del trabajo ya hecho (adultos mayores retirados del mercado de trabajo) ${ }^{2}$. No obstante, estas no son las únicas razones que articulan la política social y que permiten a los sujetos pertenecer legítimamente a los grupos "dependientes" de la sociedad; pues, por ejemplo en sociedades en guerra como la colombiana, la posición estructural de los sujetos respecto de la guerra (por ejemplo, las víctimas y los victimarios, sean estos efectivos o potenciales) define distintas razones socialmente reconocidas como fundamento de intervenciones sociales del Estado.

De esta manera, al momento de pensar una política social cualquiera, es importante lograr explicitar cuáles son los contenidos políticos de los que son portadoras distintas propuestas de intervención social, pues las diferentes "razones" que habilitan intervenciones sociales del Estado legítimas, definen las características de ese Estado y de la sociedad que lo constituye. Al respecto, no es ninguna novedad decir que la gobernabilidad y la legitimidad tienen altas deudas con la política social, sino que lo que se debe saber es cómo se da esta relación, pues no es lo mismo si la legitimidad se produce, por ejemplo, apelando a discursos que se erigen en términos de derechos, de necesidades, de libertad de elegir, de responsabilidad, de esfuerzo individual, que si se produce por la proliferación de discursos cuyos puntos nodales son, por ejemplo, significantes como seguridad, orden o paz ${ }^{3}$.

2 Al respecto, ver el proyecto Ubacyt (2011_1014). "Autovalía y dependencia legítima en las políticas sociales de Argentina del segundo centenario (1910-2010)". Dirigido por Estela Grassi. En: $<$ http://www.webiigg.sociales.uba.ar/grassi/investig7.html>

3 Todos los discursos señalados (derechos, necesidades, seguridad, orden, etc.) y las intervenciones sociales que generan, son portadoras de legitimidad, pero los modelos de legitimidad que los construyen y que estos reproducen son distintos, pues estructuran diferentes cadenas equivalenciales
En ese sentido, es importante incorporar a los análisis sobre modelos de política social, las características de su legitimidad, lo cual supone preguntarse específicamente por a) cuáles son los sujetos que cada modelo de política social construye y b) cuáles son las razones que construyen el merecimiento de dichos sujetos. De modo que, "el pobre" como destinatario, "el trabajador" como destinatario, "el ciudadano" como destinatario, serían los tres protagonistas más clásicos (en clave de Esping Andersen, 1990), pero en este artículo se propone considerar la posibilidad (y la necesidad) de pensar también a "sujetos liminares" como "las víctimas", a "los victimarios" y a "la población en situación de riesgo" de ser afectada por la violencia de distintos grupos armados, como legítimos destinatarios en un modelo de política social neoliberal que se construye al pulso del acontecer de la guerra. Esto no implica desconocer que en toda sociedad capitalista, la paz y la seguridad se establecen como puntos nodales, en torno a los cuales se definen las intervenciones sociales del Estado, sino que exige profundizar en la idea de que en los países en guerra prolongada, como Colombia, muchas intervenciones sociales del Estado, al definir su población por sus condiciones estructurales respecto a la guerra, se conforma un nuevo tipo de sujeto de política social: "el sujeto liminar" o "sujeto de la violencia", conformado por grupos de individuos que actúan la violencia, que la sufren o que están en riesgo de actuarla. Legítimos destinatarios de políticas sociales, que se construyen al pulso del acontecer de la guerra.

En las sociedades capitalistas, en las que la mayoría de las personas fundan sus relaciones $y$ organizan su vida alrededor de su participación en el mercado laboral, la política social se ha desplegado como aquella intervención del Estado que "condensa el sentido de la acción estatal en la reproducción de la vida" (Danani, 2008: 29), al producir y moldear directamente las condiciones de la vida y de reproducción para distintos grupos sociales que dependen de sus ingresos laborales. Esto lleva a recordar que

(Laclau y Mouffe, 2005) que definen como legítimas algunas demandas sociales y excluyen otras. 
no todos los grupos sociales son construidos al mismo tiempo como población-objetivo o destinatarios de política social y hace necesaria la pregunta por las características que van asumiendo para serlo y de qué modo se da este proceso.

Estas características de los grupos y sujetos beneficiarios/destinatarios de programas y planes sociales, son definidas históricamente a partir de la tensión (siempre particular) que amenaza la integración de una sociedad (de manera inminente o potencial) y que se busca "conjurar" (Castel, 1995) ${ }^{4}$. Así, el "merecimiento" resulta entonces una categoría fundamental para este análisis, porque construye la población-objetivo de una política; pero a la vez sumamente problemática, porque responde a criterios siempre contingentes $y$ de diferente naturaleza. Es decir, "merecimiento" es una noción históricamente situada que determina la construcción de la población beneficiaria y que como categoría analítica, debe ser contextualizada.

De esta manera, la constitución de una población objetivo o destinataria de una política social, debe ser analizada como un proceso en el que intervienen distintos vectores de atribución de sentido que adquieren identidad a la luz de la específica correlación de fuerzas que los construyen.

El otro vector desde el cual se propone desarrollar este análisis sobre las políticas sociales en Colombia, comprende estas políticas como el resultado de intercambios y entrecruzamientos entre múltiples agencias con capacidad y efectos políticos distintos, y no como el fruto de la voluntad unilateral del gobierno. Es decir, la política social debe ser valorada

4 Danani (2008: 42) señala que "ni la pobreza, ni la condición familiar o laboral, $y$ ni siquiera la condición de ciudadano han sido estatus que funcionaran como salvoconductos automáticos [que habiliten a determinados sujetos como beneficiarios de una política social, sino que estos] siempre se han visto redefinidos por otras condiciones. El caso tal vez más claro es el de la pobreza: nunca bastó con ser pobre para "ser merecedor" de ayuda (sea lo que fuere que eso signifique): "pobre merecedor" no es el pobre a secas, sino aquel que demuestra que ha hecho todo lo posible por salir de la pobreza, principalmente, trabajar". como un escenario de disputas, sin desconocer que, en definitiva, son los organismos estatales los que tienen prioridad lógica e histórica de incluir un tema en la agenda política (lo cual implica la capacidad de definir y reconocer problemas $y$ necesidades). Es entonces importante comprender el proceso de discusión y gestión de la política social como un momento en el que distintas agencias de poder luchan por conservar o por renovar distintos derechos e intereses; es decir, como un "momento" de constitución legítima de las normas.

A menudo, los análisis de políticas sociales se desarrollan en torno a dos formas de intervención que son, en primer lugar, la desmercantilización de bienes y servicios, y en segundo lugar, la desmercantilización de las personas. Este proceso de desmercantilización es definido por Esping Andersen (1993: 41) como el proceso por el cual la prestación de algunos servicios se torna una cuestión de derecho (y no de "compra" o función de la capacidad económica). Desmercantilizar implica entonces que el acceso a ciertos bienes y servicios (de todos, o de al menos algunos grupos sociales) no sea objeto de regulación por el mercado. Pero esta sustracción del mercado no es un proceso amplio o descontextualizado $y$ a partir del cual las personas que dependen de sus ingresos laborales, se liberan de la necesidad de vender su fuerza de trabajo; más bien, es un proceso de alcances específicos y limitados, a partir del cual una sociedad determinada conjura su cuestión social. Siguiendo esta línea teórica, la cuestión social ha sido entendida como una aporía fundamental en la cual una sociedad experimenta $y$ contiene la amenaza de su fractura en las sociedades capitalistas contemporáneas, esa amenaza estructural casi siempre se funda en la relación capital/trabajo (Castel, 1995).

En el caso colombiano, dado que la cuestión social se configura históricamente y de manera particular en cada sociedad, la amenaza que experimenta la sociedad responde a un momento lógico anterior a la configuración problemática de la relación capital/trabajo, pues es la guerra el elemento que, de manera permanente, pone a la sociedad colombiana al borde de su ruptura. Esto implica que la política 
social busque, dentro del marco aporético de la relación capital/trabajo, construir las condiciones de vida de los sujetos "tradicionales" de la política social ya mencionados, pero además, incluya intervenciones encaminadas a incorporar al núcleo de la sociedad a sujetos que a) actuaron la violencia de modos que amenazan la existencia de la sociedad (excombatientes); b) son testimonio de la ruptura de los límites de la sociabilidad que implica la violencia armada ilegal (víctimas) y c) viven en condiciones sociales que los hacen más propensos a desconocer las condiciones fundamentales de la sociabilidad (población en alto riesgo). Para ello articula distintas estrategias de normatización y normalización por las que el Estado interviene en la producción de las condiciones de vida de los sujetos asistidos, a partir de la capacitación laboral y el mandato del autovalimiento; pero también y fundamentalmente, radicando en ellos las normas y prohibiciones fundamentales de la sociedad, a partir de los dispositivos de acompañamiento psicosocial.

En otras palabras, la presencia de la guerra en el modelo de política social colombiano se hace visible en el momento en que el Estado colombiano contempla leyes, planes, programas, partidas presupuestarias y organismos específicos de implementación para distintas intervenciones que, sin salir del marco aporético de la relación capital/trabajo, buscan generar estrategias de normalización y normatización, capacitación, autovalimiento, asistencia, por medio de las cuales construir las condiciones de vida de la población, cuya existencia ha sido definida por el enfrentamiento violento de estructuras armadas (legales e ilegales, entre ellas y contra el resto de la población).

Es claro pues, que la política social no existe de manera prefigurada o genérica $y$ común a todas las sociedades; por el contrario, sus acciones son construidas, propuestas y llevadas a cabo, respondiendo a las tensiones sociales, económicas, políticas específicas y únicas de cada sociedad; las cuales están construidas $y$ determinadas por intereses $y$ necesidades de muy diversa índole.

A partir de estas claves de lectura: la política social como un proceso sociohistó- ricamente determinado de definición y contención de la cuestión social, y con ello de definición de las dimensiones, condiciones, discursos y razones de la asistencia, se analizarán cuatro maneras en las cuales se observa que la guerra afecta de manera directa la política social colombiana. Estas son, ordenadas de mayor a menor abstracción: a) radicalizando su protagonismo en el proceso de ordenamiento simbólico de la sociedad; b) construyendo sus sujetos; c) creando sus problemas $y$ d) definiendo su agenda y su presupuesto. A continuación, se desarrollarán brevemente cada uno de estos puntos.

\section{A) RADICALIZANDO SU PROTAGONISMO EN EL PROCESO DE ORDENAMIENTO SIMBÓLICO DE LA SOCIEDAD}

Para desarrollar esta dimensión es preciso plantear, aunque sea de manera breve, algunas cuestiones fundamentales que hacen a la comprensión de la sociedad como ordenamiento simbólico. En este sentido, es necesario entender la sociedad como la articulación de distintos afectos, intereses, sentidos individuales $y$ colectivos, organizados en torno a unos principios ordenadores que permiten establecer una economía afectiva y representacional, creando su estructura $y$ permitiendo su reproducción. Estos principios ordenadores que funcionan como marco y guía del accionar de grupos y sujetos, se presentan bajo la forma de prohibiciones fundamentales, las cuales establecen las fronteras entre lo social (como mundo de creencias $y$ deseos individuales $y$ colectivos no ligados, no ordenados) y la sociedad. En otras palabras, definen los contenidos de lo que será socialmente considerado debido o indebido, bueno o malo, prohibido o permitido, legal o ilegal. Es decir, establecen los límites entre aquello que pertenece propiamente a la sociedad y la organiza en su interior, $y$ aquellos elementos que desde dicho ordenamiento se rechaza y deben ser excluidos. Estas exclusiones a partir de las cuales se erige la sociedad, este establecimiento de límites entre la interioridad y la exterioridad societal, tienen la forma de 
prohibiciones fundamentales ${ }^{5}$. 0 dicho de otra manera, el establecimiento de un límite social construye performativamente a determinados actos y determinados sujetos como opuestos, por grado o por signo, a las premisas básicas de la vida en sociedad: el respeto a la vida, el cuidado del cuerpo, la reproducción de la vida por medios legales, son solo algunas de una larga lista.

En este sentido, los individuos se forman como sujetos sociales, a partir de la radicación de estas premisas, pues ellos también son, al igual que lo social, campos de permanente flujo $y$ exceso de deseo que requiere ser ligado, ordenado en un sistema de diferencias. Todo esto equivale a decir que, tanto la sociedad como el sujeto social, solo existen en tanto sistema de diferencias $y$ en tanto sistema de clasificación cognitiva y axiológica de lo que es y lo que debe ser. Violentos serían entonces aquellos actos que rompen este ordenamiento, que desconocen estas prohibiciones y estas premisas, que contradicen el código y credo societal.

Ahora bien, si se acepta que la prohibición del uso de la violencia física, por parte de individuos o grupos no estatales, es uno de los principales ordenadores sociales, se debe admitir que los estados de guerra interna son situaciones en las que los sujetos que protagonizan esa violencia (como actores o receptores de esta) ponen en vilo la existencia misma de la sociedad. Son testimonio del exceso, de la violación de las reglas fundamentales de toda sociabilidad. En otras palabras, los sujetos de la violencia son manifestación de la puesta en cuestión de lo incuestionable; lo que es equivalente a decir que dan cuenta de la inestabilidad del ordenamiento social y dando un paso más, se puede decir que son testimonio de que cualquier prohibición, aunque sea fundamental, es susceptible de desconocimiento. Son, en la clave de lectura propuesta: "sujetos liminares".

Con el fin de reprimir, diferenciar y conjurar estas prácticas violentas que pueden propagarse y producir estados de indiferenciación

$5 \quad$ Se entiende por prohibiciones fundamentales, aquellas prescripciones políticamente establecidas $y$ de obligatoria observancia, sin las cuales no es concebible la vida en sociedad. social, se establecen instituciones y mecanismos de intervención, cuya finalidad es restablecer el ordenamiento simbólico y radicar nuevamente en los individuos las prohibiciones fundamentales. El sistema penal es uno de estos mecanismos, pero como seguramente ya sospecha el lector, no es el único. Es momento de volver a hablar de la política social colombiana.

En este punto del artículo, comienza a tener más sentido la propuesta de trabajar con una noción de política social que la valora en su carácter de forma de producción de la vida individual y societal. Es decir, como intervención estatal cuya tarea principal es producir (y reproducir, sin que ello implique "repetir") las condiciones de vida de los sujetos y en ese proceso, hacer sociedad al mismo tiempo que los sujetos alcanzados por ella son construidos. Así, toda política social participa de la construcción de la sociedad como ordenamiento simbólico, en tanto prescribe formas de vida $y$ de reproducción de la vida, pero en el caso colombiano esta participación es radical; esto es, mucho más profunda y visible porque actúa sobre sujetos y fenómenos cuya presencia y accionar constantemente cuestiona este ordenamiento en su carácter de sistema cognoscitivo $y$ axiológico.

A modo de ejemplo, se tomarán tres casos de programas que permitirán apreciar más claramente cómo se da este proceso de radicalización de la intervención de la política social en el ordenamiento simbólico. La presentación que se hace de estos programas es sumamente breve porque el objetivo no es exponerlos, sino solo usarlos como referencias para el análisis. Estos son:

$\diamond \quad$ Los programas dirigidos a excombatientes de grupos armados, como es el caso del Programa de Atención Humanitaria al Desmovilizado (PAHD), cuyo objetivo es lograr la integración social de sujetos que participaron en grupos armados al margen de la ley, a través de su autovalimiento en la legalidad. El PAHD además de una asignación monetaria mensual, brinda a sus beneficiarios capacitación e 
inserción laboral, vinculación del excombatiente y su familia al régimen subsidiado de seguridad social, atención y seguimiento psicosocial, por un lapso no menor a 18 meses.

$\diamond \quad$ Los programas de atención a las víctimas del conflicto armado, implementados a partir de la Ley 975 del año 2005, los cuales a través de reparaciones económicas y simbólicas, acompañamiento jurídico y psicosocial, vinculación al régimen subsidiado de seguridad social, capacitación e inserción laboral, entre otros beneficios, buscan la autovalía de las víctimas, el restablecimiento de sus derechos $y$ el reconocimiento de su dignidad.

$\diamond \quad$ Los programas dirigidos a población en riesgo de ingresar a formar parte de grupos armados, como los programas Jóvenes en Alto Riesgo (JAR) y Jóvenes con Futuro (JF), que a través de capacitaciones para el trabajo, vinculación laboral, asignaciones monetarias $y$ asistencia psicosocial, entre otros beneficios, buscan contribuir a la reducción de posibilidades de que los jóvenes se vinculen en actividades al margen de la ley.

Como primera cuestión, se debe señalar que los tres tipos de programas usados a modo de ejemplo, se plantean como dispositivos de inserción laboral. Esto es, intervenciones sociales cuya finalidad es, entre otras, que los beneficiarios adquieran capacidades $y$ destrezas que los habiliten para ser competentes laboralmente y así, proveerse sus medios de vida de manera autónoma.

Al plantearse así el problema, la competencia laboral es un requisito de la integración. De allí que una de las finalidades de estos programas, sea desarrollar individuos aptos para la convivencia en sociedad y acordes a las exigencias del mercado laboral, es decir, se proponen construir a los beneficiarios como sujetos empleables. Así, las actividades desarrolladas por estos programas pretenden dar forma a distintos momentos de un proceso, en el que se involucran saberes $y$ destrezas, pero también actitudes $y$ disposiciones de los beneficiarios.
Si bien, puede parecer redundante o inferirse de la definición y el tratamiento que en este artículo se le ha dado a la política social, debe remarcarse que el control social es subyacente a toda intervención estatal; es decir, que "cuando el Estado entrega servicios de salud, previsión, educación, entrega no sólo servicios materiales" (Lechner, 1997: 5) y en esa medida, programas como los señalados, no son simplemente un instrumento de inserción laboral de excombatientes, víctimas y jóvenes potencialmente delincuentes, sino que su implementación va dirigida a instituir la relación simbólica del Estado como ordenador y garante de la vida en común, a través de la construcción de las condiciones de vida de los sujetos beneficiarios y principalmente, a partir de la radicación en estos, de las prohibiciones fundamentales y criterios axiológicos de la vida en sociedad, por medio de sus principales componentes, como se verá a continuación. En otras palabras, conectando con lo expuesto anteriormente, en este artículo la política social no está definida por sus acciones compensatorias o protectoras, pues esto sería reducirla y considerarla como una "reacción", cuando lo que se intenta resaltar es su carácter "constructor" de sujetos y de sociedades.

Vale la pena recordar que este proceso de construcción de condiciones y capacidades que desarrollan estos programas, asume múltiples formas, entre ellas, como mecanismos de seguimiento y normalización de las conductas del beneficiario en su círculo familiar, escolar, laboral y comunitario. Buscan en todo momento instalar en los sujetos, el sentido de lo bueno $y$ lo desviado, lo socialmente valorado como correcto y lo permitido. Así, esta construcción del sujeto es un proceso que se da a través del desarrollo de distintas estrategias y componentes que construyen socialmente una definición de los valores de justicia, igualdad, desigualdad, etc. Estos se despliegan simultáneamente en los componentes de asistencia socioeconómica y psicosocial, los cuales, a partir de la capacitación para el trabajo y el acompañamiento psicológico familiar e individual, junto a la búsqueda de la inserción laboral de los excombatientes, constituyen una intervención social del Estado 
que lo contiene y cuya meta principal es pacificar la sociedad, domesticar la violencia.

De esta manera, la intervención social del Estado en las condiciones de reproducción de la vida de los sujetos más cercanos estructuralmente a la dinámica de la guerra, debe ser comprendida en un nivel de referencia distinto al que normalmente está presente en los análisis de política social. Así, una particularidad del caso colombiano, es que en él muchos programas intervienen, previenen y conjuran la amenaza de ruptura de la sociedad, a partir de la construcción de las condiciones socioeconómicas de determinados sujetos, pero no por la situación deficitaria de las condiciones y los sujetos, sino porque estas y estos son socialmente considerados un núcleo de gestación de la violencia. Encarnan la amenaza y la evidencia de que otras formas de vida son posibles. La amenaza conjurada debe entenderse entonces en el sentido mas primario de la expresión, pues es la "capacidad de fuego" de los excombatientes la que incide en su construcción como población beneficiaria, la ruptura al orden que supone el testimonio de las víctimas si su dignidad no ha sido reparada, la cual las construye como población con necesidades específicas para el Estado y la potencial capacidad de fuego de la población en riesgo, lo que finalmente los construye como sujetos legítimos de política social. Lo anterior permite avanzar al segundo punto.

\section{B) CONSTRUCCIÓN DE SUS SUJETOS}

Otro de los ámbitos en que la guerra define las características de la política social es en la construcción de los sujetos de esta última. Esta construcción se da en dos modalidades distintas, una de carácter constitutivo o preformativo: 1) definiendo los sujetos de la intervención y otra de carácter más práctico, ligada a la incidencia de la guerra sobre el "hacer" de la política social: 2) definiendo los sujetos de la gestión.

1) DEFINIENDO LOS SUJETOS DE LA INTERVENCIÓN

Con lo expuesto hasta ahora se puede concluir que dos de las características más importantes del caso colombiano son: su noción compleja de merecimiento y su escisión radical entre población destinataria y población protegida.

Muchos de los programas implementados en las últimas décadas en Colombia, intervienen $y$ en la mayoría de casos mejoran, las condiciones de vida de los sujetos beneficiarios; sin embargo, los fundamentos, la presentación $y$ la justificación de estos, indican que su finalidad principal es proteger a "la sociedad" en su condición de sociedad amenazada por la guerra. Es cierto que, en términos generales, toda política social puede ser leída como una protección e intervención de la sociedad sobre sí misma (Castel, 1995), pero en el caso de lo que tentativamente en este artículo se nombrará como "política social de guerra", la distinción entre población "asistida" y población "protegida" está mucho mas marcada. Así, constantemente, las intervenciones sociales del Estado colombiano se despliegan bajo la forma de planes $y$ programas, cuyo principal fundamento es que mejorar las deficientes condiciones socioeconómicas de determinados grupos sociales es necesario porque ello representa el mejoramiento para la calidad de vida $y$ tranquilidad del resto de la sociedad, de los sujetos que no participan ni testimonian los límites de la sociedad. Así, se genera una división que no es puramente analítica entre a) población asistida y b) población protegida por la política social.

Los sujetos que componen el primer grupo, son sujetos construidos como beneficiarios legítimos de política social por el lugar que ocupan en el desarrollo de la guerra: los límites de la sociedad, las fronteras de construcción del ordenamiento simbólico. Son entonces "sujetos liminares" que por sus condiciones socioeconómicas, también pueden ser considerados "sujetos pobres". Pero esta última condición no es la que los define como objeto de política social, pues la razón que finalmente los habilita y los construye como beneficiarios es que son sujetos que actúan la violencia, la sufren o potencialmente la actuarán.

Por otra parte, los sujetos que componen el segundo grupo, la población protegida, claramente no son los mismos beneficiarios 
de estos programas, pero aún así pueden ser considerados sujetos de la política social porque son su razón de ser: es en aras de la protección de estos que se asiste a los primeros. Es decir, construyendo las condiciones de vida de los sujetos que actúan o que potencialmente actuarían y testimonian la violencia, se protege a los demás ciudadanos de la agresión violenta y a toda la sociedad como ordenamiento simbólico.

Así, las políticas sociales construyen nociones socialmente aceptadas de lo normal y lo no normal, a partir de la definición de sujetos $y$ grupos sociales como merecedores, $y$ las razones que habilitan dicho merecimiento.

Como ya se señaló, para el caso colombiano es necesario elaborar una noción compleja de merecimiento, que lleve al extremo su carácter problemático y sociohistóricamente determinado, distinguiendo entre las características individuales de la población merecedora $y$ las condiciones sociales en que el programa interviene. Anteriormente, se planteó como una clave de lectura, analizar cómo se construye un modelo de política social a partir de razones distintas a la problemática relación capital/trabajo. En esta medida, la noción de merecimiento que socialmente se construye frente a programas como los mencionados (de atención a excombatientes, víctimas y jóvenes en alto riesgo) es fundamental en este planteamiento, pues posiciona como fundamentos de legitimidad de la asistencia a significantes como seguridad $y$ orden.

En otras palabras, en contextos límite como el colombiano, el merecimiento debe ser comprendido al margen de cualquier contenido que a priori, califique a una persona como alguien que "tiene" aquello que "debe tener moralmente $e^{6}$ " $y$ debe tener en cuenta argumentos que construyen una noción de merecimiento compleja, en la que intervienen otros intereses, como por ejemplo, la conveniencia social de la intervención estatal.

En este sentido, se puede por ejemplo, hacer la lectura del PAHD como un dispositivo social del Estado que busca sacar a los excombatientes de la lógica de la guerra, a través de

6 Sean estas cualidades buenas o malas, porque también existe el "merecimiento" de castigos, o la penalidad merecida. la generación de capacidades, bajo el supuesto de que la puesta en juego de estas en el mercado de trabajo legal, permitirá satisfacer sus necesidades cotidianas; pero siempre teniendo en cuenta que el interés en el autovalimiento de los excombatientes, su definición y reconocimiento como población merecedora de esta asistencia, radica en el impacto que el cumplimiento de este objetivo tiene sobre aquello que hegemónicamente se defina como "seguridad" u "orden".

De esta manera, estos programas definidos por las condiciones de la guerra, desmercantilizan algunos bienes y servicios para el beneficio de su población asistida, pero también configuran formas específicas de control y disciplinamiento social, que pueden perfectamente ser estudiadas desde el campo específico de la política social. En este caso, la población que es objeto de ese control son sujetos peligrosos, potencialmente peligrosos o que testimonian la violencia y que para el Estado merecen ser asistidos, porque ello afecta positivamente la vida cotidiana de los demás ciudadanos, al brindarles mayor "seguridad" y restablecer el "orden" de la sociedad como ordenamiento simbólico.

\section{2) DEFINIENDO LOS SUJETOS DE LA GESTIÓN}

Si en el apartado anterior se analizó cómo la guerra establece discursos y razones que construyen a determinados grupos sociales como nuevos sujetos de las intervenciones sociales del Estado, en este apartado interesa ver cómo este mismo conflicto incide en la implementación de dichas intervenciones. La tipología elaborada por Velásquez $(2009 a)^{7}$ será un insumo principal en esta descripción. A partir de esta, se puede sostener que existen dos modalidades con las cuales la guerra define los sujetos de la gestión: incidiendo "desde afuera" e incidiendo "desde adentro" de la gestión de la política social.

$7 \quad$ La mayoría de la información utilizada para este punto (Control de la Gestión) está tomada de Velásquez (2009). Las otras caras del poder: territorio, conflicto y gestión pública en Colombia. Editado por Foro Nacional por Colombia, RINDE y la Agencia de Cooperación Alemana en Colombia, GTZ. 
Lo que Velásquez (Ibid.) denomina la incidencia externa, es un fenómeno producido por el accionar de los grupos armados al margen de la ley, que implementan prácticas como el "veto territorial", con el fin de impedir que determinados recursos lleguen a zonas de su dominio y establecen nuevas directrices, según las cuales la realización de acciones por parte de las administraciones municipales tienen que contar con su aprobación. La segunda modalidad que interesa resaltar de esta incidencia externa, se ejerce a través de la presión a las autoridades municipales para que orienten la inversión hacia zonas de influencia de los actores armados o para que contraten personas $y / 0$ entidades de su confianza.

Por su parte, lo que se denomina "incidencia interna" tiene también, al menos, dos modalidades de ejercicio. Una de ellas es la captura de rentas, la cual consiste en la asignación de cargos y/o contratos a personas (naturales $y$ jurídicas) afines a distintas organizaciones armadas, con lo que estos grupos se aseguran la apropiación de un porcentaje del valor total de los proyectos implementados; a través de esta modalidad de incidencia, los grupos armados también consiguen extorsionar a contratistas, los cuales, si quieren realizar las obras para las que fueron contratados deben destinar un porcentaje de su ganancia a cambio del permiso para trabajar en las zonas de dominio ilegal. Esta modalidad de incidencia también se expresa en otras prácticas, tales como, la creación de empresas de prestación de servicios, la contratación de testaferros de los actores armados para la ejecución de obras públicas o incluso, la presencia efectiva de las organizaciones armadas en la gestión de las políticas sociales, a través de la contratación de miembros como funcionarios de entidades públicas (Velásquez, 2009a).

La segunda modalidad con la cual la guerra incide internamente en la gestión de la política social, es a partir de la definición de los contenidos de los planes y programas sociales. Anteriormente, se expuso brevemente cómo los discursos de orden y seguridad son el fundamento de programas de reinserción, reparación $y$ prevención de distintas manifestaciones $y$ actores de la violencia, pero en este punto, el análisis se plantea en un registro diferente, más vinculado con la gestión de los programas que con su construcción. En esa medida, los actores armados definen el contenido de planes y programas también por medio de la cooptación de distintos niveles de representación política, desde locales hasta nacionales, que logran impulsar la aprobación de planes, programas $y$ proyectos en los cuales los actores armados tienen principalmente, intereses económicos.

\section{3) LIMITANDO SU CAPACIDAD DE EJERCICIO DEL PODER POLÍTICO}

Una tercera modalidad. Anteriormente, se propuso comprender la política social como un escenario de disputas entre agentes con distintas capacidades $y$ una desigual distribución del poder. Se le nombró además, como un momento de constitución legítima de normas. Ahora bien, este proceso de lucha política y construcción normativa de la sociedad es radicalmente modificado $y$ no puede considerarse democrático cuando algunos de sus agentes legítimos de poder político no gozan de las mínimas garantías de lucha y supervivencia. En este sentido, no es necesario profundizar en descripciones para exponer de qué manera la guerra limita (cuando no anula de raíz) la capacidad de representación y demanda de múltiples actores populares. Baste señalar que en Colombia, entre 1986 y 2006, han sido asesinados 2515 sindicalistas y entre 1974 y 2004, ha habido 6745 violaciones de derechos humanos contra las poblaciones indígenas, de las cuales más de 2000 han sido asesinatos.

Otro dato clave para pensar en la fuerza de negociación y de construcción de las políticas sociales y laborales, es que actualmente en Colombia, el porcentaje de sindicalización es menor al 5\% y que los lugares más conflictivos y más productivos del país, son los mismos en los que más ha declinado la protesta social a manos de las dominaciones irregulares ${ }^{8}$. Al

8 "Para el total de la muestra [basada en la EPH] se obtuvo que la tasa de densidad sindical es de $3,4 \%$, siendo en los sectores público y privado de $21,9 \%$ y 1,4\%, respectivamente. Esta gran diferencia entre sectores está relacionada con las características especificas de los ocupados como 
respecto, señala Sarmiento Anzola que "en 1990 la proporción de trabajadores sindicalizados era del 12,8\%, en 1997 había caído a 6,5\% y en 2009 apenas supera el 3\%, con la característica de una alta fragmentación en sus organizaciones" (2009: 72).

\section{C) CREANDO LOS PROBLEMAS}

El conflicto armado es un factor de construcción de condiciones de vida, pues define $y$ modifica el contexto y las condiciones de reproducción de los sujetos, alterando trayectorias vitales, laborales, educativas, trayectorias de inserción $y$ reconocimiento social, etc. En este sentido, existen varios tipos de problemas producidos por la dinámica de la guerra y que son campos de directa incumbencia para la política social, pero que no tendrían lugar si las condiciones de la guerra no estuvieran presentes en la sociedad.

Solamente en las últimas dos décadas, la guerra ha generado el desplazamiento de cerca de cuatro millones de campesinos hacia grandes y medianos centros urbanos. Estos sujetos desplazados, hijos directos de la guerra, pasan de tener sus necesidades básicas satisfechas a vivir en condiciones de pobreza extrema y con un nivel de activos, ingresos y educación menor que el de los pobres urbanos ${ }^{9}$.

Otro caso paradigmático de una problemática de necesaria intervención desde la política social cuyas características son estrictamente definidas por la guerra, es el de las

nivel de escolaridad y experiencia, duración del contrato de trabajo, tamaño de firma, entre otros. $\mathrm{Al}$ estimar estos mismos indicadores considerando los ocupados asalariados con nivel educativo inferior a universitario completo, que en adelante será la población objetivo, se obtiene que la tasa de densidad sindical es de 1,8\% para el total y de $1,05 \%$ y $14,77 \%$ para los sectores privado y público respectivamente" (Guataquí et ál., 2009).

Desde 1997 a diciembre de 2009, según el gobierno en Colombia, han sido desplazados 747719 hogares, con un total de 3292666 personas. Sin embargo, según CODHES (Consultoría para los Derechos Humanos y el Desplazamiento), la cifra supera los 4,2 millones de personas. Los años más críticos de desplazamiento fueron el 2000, 2001 y 2002, que a su vez son los años de más intensa actividad de los grupos guerrilleros y paramilitares. desmovilizaciones de excombatientes, los cuales al igual que las víctimas, también son consecuencia del conflicto. Esta población, creciente a medida que se producen más desmovilizaciones colectivas e individuales, responde a características específicas que ya se abordaron anteriormente, suponiendo para el Estado y la política social, la creación de programas de asistencia y seguimiento para cerca de 50000 excombatientes y sus familias en concepto de salud, educación, ingresos $y$ asistencia psicosocial.

Otro punto, pocas veces valorado por los analistas de políticas sociales en Colombia, entre otras razones por la escasa información existente al respecto, tiene que ver con que durante un conflicto armado interno "la tasa de mortalidad infantil se incrementa en un 13\%, cifra que después de cinco años de terminado el conflicto se encuentra en un $11 \%$ superior al valor preconflicto" (Ryan, 2005, tomado de Valencia, 2006: 162), lo cual abre un interrogante de necesaria investigación, que no anula el problema sino que le agrega complejidad y que tiene que ver con la definición de las cifras de mortalidad infantil "preconflicto", en una sociedad que durante más de cuarenta años ha vivido en medio de este ${ }^{10}$.

\section{D) DEFINIENDO LA AGENDA Y EL PRESUPUESTO}

Además del protagonismo que la guerra le otorga a la política social en la construcción

$10 \quad$ Otro punto clave para tener en cuenta en este análisis, pero en el que por cuestiones de extensión y complejidad no se profundizará, tiene que ver con el incremento de la desigualdad y su íntimo vínculo con la concentración de la riqueza de la tierra, concentraciones que a su vez están en relación directa con la dinámica espacial y económica del conflicto armado (Reyes, 2009). Así, en Colombia, según Sarmiento Anzola (2009: 72) en los últimos 25 años "la gran propiedad (más de 500 hectáreas) pasa de controlar $47 \%$ a $68 \%$ de la superficie catastrada; entre tanto, la pequeña propiedad pasa del $15 \%$ a $9 \%$. Las fincas menores de 3 hectáreas pertenecen al $57,3 \%$ de los propietarios; en contraste las fincas con más de 500 hectáreas corresponden al $0,4 \%$ de los propietarios. En Colombia, 13000 personas naturales son dueñas de 22 millones de hectáreas" [algo así como 1693 hectáreas por cabeza]. 
del ordenamiento simbólico de la sociedad, las distintas formas de definición de los sujetos de la política social, la responsabilidad de la guerra en la producción de problemas objeto de política social, una cuarta y última dimensión en que la guerra construye a la política social en Colombia, tiene que ver con el protagonismo que tiene la primera en la definición de la agenda y el presupuesto de la segunda.

Esta incidencia puede ilustrarse del siguiente modo: según el artículo 366 de la Constitución Nacional de Colombia:

... el bienestar social y la mejor calidad de vida de la población son finalidades sociales del Estado y el objetivo fundamental de su actividad será la solución de las necesidades insatisfechas en salud, educación, saneamiento ambiental y de agua potable. Para tales efectos, en los planes y presupuestos de la nación $y$ de las entidades territoriales, el Gasto Público Social tendrá prioridad sobre cualquier otra asignación (Art. 366).

Sin embargo, el artículo 350 de la misma carta señala que esta distribución del gasto puede modificarse "en los casos de guerra externa o por razones de seguridad nacional" (Art. 350). De manera que, por esta vía de excepción planteada por el artículo 350, el presupuesto de planes, programas y proyectos de corte más "tradicional" en términos de política social, han visto seriamente comprometido su presupuesto $y$ existencia, pues el razonamiento que se ha aplicado indica que el Gasto Público Social puede ser invertido en "la solución de necesidades básicas insatisfechas de salud, educación, saneamiento ambiental, agua potable, vivienda, [pero también, en todas aquellas acciones] tendientes al bienestar general $y$ al mejoramiento de la calidad de vida de la población" (Art. 17 de la Ley Orgánica de 1994, citado por Barón Leguizamón, 2009: 146). Es decir, cuestiones coyunturales, que afectan la calidad de vida de la población, pero que responden a una matriz distinta a la de la relación capital/trabajo, como las concernientes a la seguridad y el orden público, son legítimamente valoradas como campos de acción e intervención de políticas sociales.
En este sentido, Barón Leguizamón (2009: 171) señala dos datos interesantes para análisis del caso colombiano y con los cuales se da por terminada esta exposición; primero, entre el año 2000 y el 2004, cerca del 13\% del gasto público social fue destinado a funciones de defensa y seguridad nacional; segundo, el Fondo de Protección Social, creado para atender a los grupos poblacionales mas desprotegidos en situaciones de crisis, ha tenido como objeto principal, según el Departamento de Planeación Nacional, "financiar los programas nacionales que el gobierno nacional defina como prioritarios $y$ aquellos programas y proyectos estructurados para la obtención de la paz"11.

\section{CONCLUSIONES}

A modo de conclusión, se puede decir que el objetivo del presente artículo fue exponer $y$ defender algunas ideas más o menos novedosas dentro del campo de estudios en políticas sociales, pero no guiados por un afán de originalidad, sino por la necesidad de comprender un fenómeno histórico concreto: la política social de un Estado en guerra. Esto implicó poner en tensión algunos conceptos y perspectivas analíticas, tarea después de la cual se puede decir que: la idea de la cuestión social, como aporía fundamental que contiene la amenaza de fractura de la sociedad, en Colombia, no se funda exclusivamente en la relación capital/trabajo, sino que halla sus raíces también en la cuestión de la guerra como fenómeno que pone en vilo toda idea de sociedad. Los procesos

11 Cabe señalar que el presupuesto para la guerra ha venido creciendo a un ritmo de casi un $15 \%$ anual desde 1992, más que triplicando el del crecimiento real de la economía. La tasa de crecimiento del pie de fuerza $(4,4 \%)$ ha duplicado el de la población colombiana (2\%) y los niveles alcanzados (160000 militares en el 2002, cerca de 400000 actualmente) representan cerca de un punto porcentual de la población. Dice Barón Leguizamón (2008) que para el periodo $2000-2007$, la tasa promedio de variación del GPS ha sido de $8,9 \%$, mientras que la del sector defensa y seguridad ha sido de 9,4\%. Los gastos militares en situación de paz equivalen a un $2,8 \%$ del PIB y en conflicto, estos se elevan a $5 \%$. En Colombia, actualmente, el gasto en guerra es del 5,6\%. 
de desmercantilización, a partir de los cuales esta cuestión social es "conjurada", no se apoyan solamente en razones tradicionales como la incapacidad, la adjudicación de responsabilidades domésticas, la necesidad de un aplazamiento para capacitarse antes de ingresar al mercado de trabajo y el reconocimiento del trabajo ya realizado; sino también en razones que plantean la posición del sujeto en el conflicto como un elemento clave en la construcción del merecimiento. Estas razones "no tradicionales" de merecimiento son adecuadas para el estudio del caso colombiano porque analiza una sociedad que erige la legitimidad de su Estado y su modelo de política social en discursos, que además de apelar a nociones como la de derechos civiles, necesidades básicas, libertad de elegir, responsabilidad y esfuerzo individual, también encuentra un núcleo legitimatorio sumamente activo en los señalamientos de la cuestión de la seguridad y el orden, como puntos nodales en la definición del discurso de la legitimidad. Esto no implica que el pobre, el trabajador y el ciudadano dejen de ser sujetos de política social, sino que complejiza esta triada de sujetos modélicos al agregar la figura de los "sujetos liminares" como destinatarios de políticas sociales en un Estado en guerra.

En pocas palabras, el análisis desarrollado, pese a su brevedad, permite comprender la política social como un proceso sociohistóricamente determinado que define y contiene la cuestión social y con ello, construye los sujetos destinatarios $y$ las condiciones y razones de asistencia, sin que ninguno de estos elementos (la cuestión social, los sujetos, las condiciones $y$ las razones de asistencia) puedan ser considerados estables o definidos con independencia de las tensiones sociales particulares, que desarrolla determinada sociedad. Esta idea también permite ampliar el campo de acción de la política social como estrategia de intervención social y como campo de investigación académica; además, permite rastrear algunas características de un modelo de política social en el que esta participa de manera protagónica en la construcción de la vida de los sujetos y de la sociedad como ordenamiento simbólico y finalmente, permite exponer los primeros argumentos en dirección a la construcción de una línea de investigación: "la política social de guerra".

\section{BIBLIOGRAFÍA}

Barón Leguizamón, Gilberto. "Colombia: cuenta de seguridad social". La seguridad social en América Latina y el Caribe. Centrángolo, Oscar (ed.). Santiago de Chile: CEPAL, 2009.

Bobbio, Norberto. El problema de la guerra y las vías de la paz. Barcelona: Gedisa, 1992.

Bouthol, G. El fenómeno de la guerra. Barcelona: Plaza y Janés, 1971.

Castel, Robert (1995). Las metamorfosis de la cuestión social. Buenos Aires: Paidós, 2004.

Danani, Claudia. "La gestión de la política social: un intento de aportar a su problematización". La gestión de la politica social: conceptos e instrumentos. Chiara, M. y Di Virgilio, Mercedes (eds.). Buenos Aires: UNGS/Prometeo, 2008.

Ermida Uriarte, Oscar. "La política laboral de los gobiernos progresistas". Revista Nueva Sociedad 211. Setiembre-octubre 2007. En: <http://www.nuso.org/revista. php? $n=211>$

Esping-Andersen, Gosta. Los tres mundos del Estado de Bienestar: Valencia: Edicions Alfons El magnánim, 1993.

Giraldo Ramírez, Jorge. Guerra Civil posmoderna. Colombia: Ed. Siglo del Hombre, 2009.

Guataquí, Juan Carlos et ál. "Determinantes estructurales de la sindicalización en Colombia". Serie Documentos de Trabajo 58. Colombia: Universidad del Rosario, Facultad de Economía, mayo 2009.

Laclau, E. y Mouffe, Chantal. Hegemonía y estrategia socialista. Madrid: Siglo XXI, 2005.

Le Bonniec, Yves. "Sistemas de protección social comparados: Colombia, Brasil y México". Revista de Salud Pública 4. Universidad Nacional de Colombia, Facultad de Medicina, 2002: 203-239.

Lechner, Norbert. "Especificando la política". Teoría y política en América Latina. México: CIDE, 1984. 
PNUD-DNP. Informe de desarrollo humano para Colombia. Misión de apoyo a la descentralización y focalización de los servicios sociales. Bogotá, mayo 1998.

Reyes Posada, Alejandro. Guerreros y campesinos. Colombia: Editorial Norma, 2009.

Ryan, B. "Book review. Democracy and ethnic conflict: advencing peace in deeply divided societies". Millenium Journal of International Studies 33 (3). Junio 2005: 917-919.

Sarmiento Anzola, Libardo. "Política social de la guerra”. Revista Foro 67. Abril 2009: 64-76.

Valencia, Germán. "La economía frente al conflicto armado interno colombiano,
1990-2006". Perfil de Coyuntura

Económica 8. Universidad de Antioquia, 2006: 141-174.

Velásquez, Fabio. Las otras caras del poder: territorio, conflicto y gestión pública en Colombia. Foro Nacional por Colombia, RINDE y la Agencia de Cooperación Alemana en Colombia, 2009a.

Velásquez, Fabio. "Actores armados y gestión municipal en Colombia". Revista Foro 67. 2009b: 16-28.

Fecha ingreso: 29/11/2011 Fecha de aprobación: 10/02/2012 